\title{
Revealing the Asymmetry of the Wind of the Variable Wolf-Rayet Star WR1 (HD4004) Through Spectropolarization
}

\author{
N. St-Louis \\ Département de physique and Centre de Recherche en Astrophysique du Québec (CRAQ), \\ Université de Montréal, C.P. 6128, Succ. Centre Ville, Montréal (Qc), H3C 3J7, Canada: \\ stlouis@astro.umontreal.ca
}

Received _ ; accepted _

To be submitted to the Astrophysical Journal soon... 


\begin{abstract}
In this paper, high quality spectropolarimetric observations of the Wolf-Rayet (WR) Star WR1 (HD4004) obtained with ESPaDOnS at CFHT are presented. All major emission lines present in the spectrum show depolarization in the relative Stokes parameters $Q / I$ and $U / I$. From the behaviour of the amount of line depolarization as a function of line strength, the intrinsic continuum light polarization of WR1 is estimated to be $P / I=0.443 \pm 0.028 \%$ with an angle of $\theta=-26.2^{\circ}$

Although such a level of polarization could in principle be caused by a wind flattened by fast rotation, the scenario in which it is a consequence of the presence in the wind of Corotating Interaction Regions (CIRs) is preferred. This is supported by previous photometric and spectroscopic observations showing periodic variations with a period of 16.9 days. This is now the third WR star thought to exhibit CIRs in its wind that is found to have line depolarization. Previous authors have found a strong correlation between line depolarization and the presence of an ejected nebula, which they interpret as a sign that the star has reached the WR phase relatively recently since the nebula are thought to dissipate very fast. In the cases where the presence of CIRs in the wind is favoured to explain the depolarization across spectral lines, the above mentioned correlation may be indicating that those massive stars have only very recently transited from the previous evolutionary phase to the WR phase.
\end{abstract}

Subject headings: Wolf-Rayet stars, Asymetric Winds, Corotating Interaction Regions, Spectropolarization, Line Depolarizarion 


\section{The Structure of Massive-Star Winds}

The phenomenal mass-loss from massive stars from their birth to their death is a crucial element of our understanding of the ecology of galaxies. The incessant exchange of gas between stars and the interstellar medium (ISM) is a determining factor of galaxy evolution, and massive stars play a particularly important role with regards to energy and momentum input, as well as in the production of chemically processed material. The amount of mass lost in each evolutionary phase but also the way it is lost, i.e. the geometry of the wind, will determine to what extent the ISM will be affected and in what way.

Equally important is what can be learned about massive stars themselves by studying their dense winds. Over the past decades, theoretical investigations on the effect of mass-loss (e.g. Maeder 1981; Chiosi \& Maeder 1986; Wooslev et al. 1993), metallicity (e.g. Meynet et al. 1994), rotation (e.g. Maeder \& Mevnet 2000; Brott et al. 2011) and magnetism (e.g. Maeder \& Meynet 2004; Meynet et al. 2011; Yoon et al. 2012) on the evolution of massive stars have been carried out (see a recent review on massive-star evolution by Langer 2012). Observationaly, we have only very limited information on the two last parameters and still have many things to learn about the first two. As the wind is initiated near the stellar photosphere, the outflow will ultimately be affected by physical processes originating at or close to the surface of the star. Therefore, studying the stellar mass-loss has the potential of helping us secure new information on physical processes for which we know very little at the present time and which have a significant impact on the life of the star. The geometry of the wind is as important a parameter as the amount of material lost. Cranmer \& Owocki (1995) and Maeder (1999) found that when gravity darkening is included, the wind driving is higher at the poles than at the equator. This will inevitably lead to a reduced loss of angular momentum and therefore of the star's rotation rate compared to the case where the wind is spherically symmetric. Nevertheless, 
Meynet \& Maeder (2000) still predict a large decrease of surface velocities on the main sequence and an even larger one when the star reaches the Wolf-Rayet (WR) phase for rotating massive stars. Any other type of wind asymmetry would have to be modelled in detail to assess how it affects the loss of angular momentum.

For WR stars, the situation is particularly unclear. At the present time, only upper limits have been set for magnetic fields in WR stars (de la Chevrotière et al. 2013). The difficulty arises because there are no photospheric lines in the spectrum of these stars and therefore we are forced to study emission lines formed in the thick wind (see predicted Stokes $V$ profiles for emission lines formed in hot-star winds threaded with a weak split monopole magnetic field in Gavley \& Ignace 2010). Constraints on magnetic fields in the wind can in principle be extrapolated back to the stellar surface, but this is very model dependent. The lack of phostopheric lines also prevents the direct determination of the rotation rate of the star by the usual method of measuring the line broadening. Indirect means have been used in the case of the presence of periodic variations in presumably single stars (e.g. Chené \& St-Louis 2010) but some uncertainties still remain.

The above-mentioned physical processes, combined with the basic radiative driving of the wind, determine its global density distribution. Initially, for simplicity, the winds were assumed to be smooth and have spherical symmetry. Not surprisingly, several observational discoveries were to prove this wrong. Photometric and spectral variations, not readily associated with easily identifiable massive $\mathrm{WR}+\mathrm{O}$ binaries, were found. To explain these, suggestions such as variability associated with the presence of a compact companion (Moffat 1982, 1983) or non-radial pulsations (Vreux 1985) were put forward but none were found to be completely satisfactory, mainly because the changes were not found to be strictly periodic.

Later, small-scale emission excesses were found superposed on the broad emission lines 
formed in the wind of several Wolf-Rayet stars (Moffat et al. 1988; Lépine \& Moffat 1999). These were eventually associated with clumps in the wind moving out radially. It is now thought that clumping is a universal parameter of massive-star winds (e.g. Lépine \& Moffat 2008). These clumps cause non-periodic photometric, polarimetric and spectroscopic variability at a relatively low level. They have led to a downward revision of the mass-loss rates of O and WR stars by a factor of 2-5 (Nugis et al. 1998; Hamann \& Koesterke 1998; Bouret et al. 2005; (St.-Louis et al. 1988), as the main observational diagnostics depend on the density squared.

With the launch of the International Ultraviolet Explorer (IUE), another observational feature that was found to be ubiquitus was the presence of Discrete Absorption Components (DACs) in the P Cygni profiles of O stars (Howarth \& Prinja 1989). Mullan (1986) was the first to point out that these features can be understood as a consequence of the presence in O-star winds of large-scale structures called Corotating Interaction Regions (CIRs), as first observed in the Sun. These spiral-shaped features are formed when a perturbation at the base of the wind, at the hydrostatic radius, causes extra radiation pressure which generates high density, low velocity regions which are then carried around by the rotation of the star (see the hydrodynamical simulations of Cranmer \& Owocki 1996). The DACs are formed when these high density zones are seen in projection in front of the star and appear as a narrow absorption feature in the major ultraviolet P Cygni absorption troughs and are found to gradually move towards higher velocities and narrower widths. CIRs are also an excellent candidate to explain optical spectroscopic variability observed in the strong emission lines of several presumably single WR stars (Dessart \& Chesneau 2002). In that case, the changes related to CIRs can be periodic but depending on the lifetime of a given CIR and the number of CIRs present, the phasing or even the general variability pattern can be different at different epochs. 
Linear spectropolarimetry of WR stars is a powerful tool to reveal global wind asymmetries. Indeed, linear polarization in these wind is readily generated from Thomson scattering of continuum light, formed near the base of the wind, off free electrons in this very hot and highly ionized environment. In a spherically symmetric and unresolved outflow, all polarization vectors exactly cancel out, but in the presence of a global asymmetry, a net continuum polarization remains. The line flux will be less polarized, if at all. Indeed, most optical emission lines are formed by recombination which produces unpolarized light. For lines that have a scattering component, some polarization can be present, but the net value will always be smaller than that of the continuum because the lines are formed further out in the wind where the density of free electrons is smaller. The further out they form, the less they are expected to be polarized. Consequently, in asymmetric winds, the relative Stokes parameters are expected to show depolarization across spectral lines.

In this paper, spectropolarimetric observations of the WN4b star WR 1= HD4004 are presented. This star was identified as a good candidate for harbouring CIRs in its wind by St-Louis et al. (2009) based on the large-amplitude spectroscopic variability it presented. Follow-up observations by Chené \& St-Louis (2010) showed that the changes were indeed periodic in spectroscopy and photometry with a period of $\mathrm{P}=16.9$ days. The kinematical characteristics of the spectroscopic variations led to the conclusion that they were generated by the presence of CIRs in the wind. Therefore, this was an ideal candidate to look for wind asymmetries via linear spectropolarization. In Section 2, the observations and data reduction procedures are presented and results are discussed in Section 3. The conclusions are given in Section 4 . 


\section{ESPaDOnS Observations and Data Reduction}

The observations in Stokes $Q, U$ and $I$ of WR 1 were obtained on September 28, 2009 at the Canada-France-Hawaii Telescope (CFHT) using ESPaDOnS1, Echelle SpectroPolarimetric Device for the Observations of Stars, which consists of a cross-dispersed, bench-mounted echelle spectrograph combined with a polarimeter. The polarization unit is composed of one fixed quarter- and two rotatable half-wave Fresnel rhombs with a Wollaston prism, which provides achromatic polarization. This high resolution ( $\mathrm{R} \sim 65000)$,

fibre-fed spectropolarimeter is designed to obtain a complete optical spectrum (from $3700 \AA$ to $10500 \AA$ ) in a single exposure.

Both the $Q$ and $U$ observations discussed in this work consist of four 650 s successive exposures in different configurations of the retarder. For the second and third position, the optics settings are changed so as to exchange the positions of the two spectra on the CCD. This allows a minimization of the systematic errors due to misalignments, transmission, seeing effects, etc. From the two sets of 4 sub-exposures, the relative polarization, $Q / I$ and $U / I$, are derived using the double-ratio method described by Donati et al. (1997).

The data reduction was carried out by CFHT staff using the Upena pipeline, which is based on Jean-François Donati's reduction package Libre-ESpRIT. Libre-ESpRIT, a self-contained package developed specifically for reducing echelle spectropolarimetric data, was first introduced by Donati et al. (1997) and later upgraded in order to be used specifically with ESPaDOnS. In this particular case, both the automatic spectrum rectification and the continuum polarization removal options have been disabled. This is because the code was not designed to work with spectra containing many strong and broad emission lines from hot star winds and will therefore not perform the data reduction

\footnotetext{
${ }^{1}$ www.cfht.hawaii.edu/Instruments/Spectroscopy/Espadons/
} 
correctly if these options are used.

The reduction of ESPaDOnS data2 begins by a geometrical analysis of each order from calibration exposures. The wavelength-to-pixel solution is obtained from a comparison frame (Thorium). From this, an optimal extraction of the spectrum is obtained. In this process, two check spectra $\left(N_{1}\right.$ and $\left.N_{2}\right)$ and error bars at each wavelength point in the spectrum are also produced. The former are null polarization profiles used to help identify spurious polarization signatures. In the final spectrum, each data point corresponds to a velocity bin of $1.8 \mathrm{~km} \mathrm{~s}^{-1}$.

Before the linear polarization analysis can be performed, a series of operations have been applied to optimize the ESPaDOnS data. First, adjacent orders were merged to produce one continuous spectrum based on the uncertainty profile supplied by Libre-Esprit. The method used is based on that introduced by Semel \& Li (1996) in which the order junctions are set at wavelengths where the uncertainty profiles from two successive orders cross each other. Note that Libre-Esprit always supplies the absolute error bars even though in our case (data reduction without continuum polarization removal) it is the relative Stokes parameters that are provided. Since the polarization level is very small, the error bar on the relative Stokes parameters is simply obtained by dividing the error bar on the absolute Stokes parameters $Q$ and $U$ by the total intensity $I$ (the exact value from error propagation should be: $\frac{\sigma}{I} \sqrt{1+\frac{Q^{2}}{I^{2}}}$ and $\frac{\sigma}{I} \sqrt{1+\frac{U^{2}}{I^{2}}}$ for $Q$ and $U$ respectively but the values of $Q$ and $U$ are very small compared to $I$ ).

The typical noise level from the raw counts of an individual velocity bin is of the order of $10 \%$. As the typical line depolarization values that have been found in the past for WR stars are of the order of $0.5 \%$, it is impossible to distinguish a signal visually. Therefore

\footnotetext{
${ }^{2}$ http://www.cfht.hawaii.edu/Instruments/Spectroscopy/Espadons/Espadons_esprit.html
} 
data binning has been performed in order to increase the $\mathrm{S} / \mathrm{N}$ of the data. As WR1 has several emission lines of different intensities in its spectrum, the signal-to-noise ratio per pixel is not constant. In order to obtain similar error bars along the spectrum, a variable binning procedure was applied as described in de la Chevrotière et al. (2013). Each final bin does not contain the same number of original bins but they all have the same error bar. No information is lost in this process if the bins are chosen to be sufficiently narrow. Indeed, for WR stars, no feature is expected to be narrower than the typical turbulence in the wind which is estimated at around $100 \mathrm{~km} \mathrm{~s}^{-1}$ (Lépine \& Moffat 1999).

\section{Spectral Line Depolarizarion in WR1}

In Figures 1 and 2, the Stokes parameters relative to the total flux (line + continuum), $Q / I$ and $U / I$, between 4400 and $7300 \AA$ are presented in the middle panels while the total flux rectified to the continuum, $I / I_{c}$, is shown in the bottom panels. The null spectra $N_{1} / I$ are presented in the top panels. The intensity spectrum presented here was generated by combining the 8 sub-exposures ( 4 for $Q$ and 4 for $U$ ) and then rectified using carefully selected continuum regions spread over the entire wavelength interval (4420-4440 $\AA ; 4770-4800 \AA ; 4901-4910 \AA ; 4975-5130 \AA ; 5540-5730 \AA ; 5940-6080 \AA ; 6735-6830 \AA)$. For shorter wavelengths, the flux level was too low to obtain small enough bin sizes to distinguish a polarization signal within spectral lines, while for longer wavelengths, the large number of telluric lines, often superposed on stellar emission lines, greatly reduces the usefulness of the polarimetric data. Here the $y$-axis for the rectified flux is truncated at 4 continuum units for clarity but the HeII $\lambda 4686$ line reaches 10 continuum units.

The signal-to-noise of the data is variable as a function of wavelength. Therefore, in this plot, a different binning of the linear polarization flux was adopted for different parts

of the spectrum. For data above $\sim 5330 \AA$, an error bar of $0.03 \%$ is used while at shorter 
wavelengths, it is $0.05 \%$, except for the HeII $\lambda 4686$ line where a value of $0.02 \%$ was adopted. Two sigma error bars are drawn in the middle panels $(Q / I$ and $U / I$ plots). Of course, the nature of the binning process is such that combining data with different bin sizes is not ideal. This can be seen at the extremities of the spectra binned to different error bars by a mismatch of overlapping bins, although they agree within the errors.

ESPaDOnS was not designed for accurate continuum polarization measurements. Tests by CFHT staff have shown that for a given star, the level of continuum polarization can vary by as much as $10 \% 3$ and that furthermore, it is not flat as a function of wavelength. However, as the change is slow as a function of wavelength, the difference between the polarization in the lines and that in the neighbouring continuum is reliable. In Figures 1 and 2, the slow trend with wavelength which has been fitted by a straight line for $U / I$ and a low order polynomial for $Q / I$ (dashed lines), is certainly instrumental. Note however that the general level of polarization detected here $(Q \sim 6.2 \% \& U \sim-1.5 \%)$ is roughly compatible with the value published by Schmidt (1988), $Q \sim 6.4 \% \& U \sim-1.1 \%$ $\left(P \sim 6.5 \% \& \theta \sim 95^{\circ}\right)$. As will be seen below, this very likely is mostly interstellar in origin.

In Figures 1 and 2, a clear signal can be seen for all main emission lines present in the spectrum of WR 1 in both linear polarization Stokes parameters. In a plot of the relative Stokes parameters as a function of wavelength, a signal from interstellar polarization would not have such a signature. Indeed, the light, regardless of its origin (line or continuum), will get polarized by the same fraction when it travels through the interstellar medium along the line of sight, yielding a flat spectrum in relative polarization. However if the continuum light from the star is polarized, then the relative Stokes parameters would show dilution of this polarized continuum by unpolarized line emission, which is exactly what is observed here. As mentioned above, for ESPaDOnS data, the actual level of continuum

\footnotetext{
${ }^{3}$ http://www.cht.hawaii.edu/Instruments/Spectroscopy/Espadons/ContiPolar/
} 
polarization is not reliable and an additional curvature caused by the instrument is observed as a function of wavelength. However, the difference between the polarization in a line and that of nearby continuum remains trustworthy. Note that the above-mentioned curvature is not to be confused with the well-known Serkowski law (Serkowski et al. 1975) describing the behaviour of interstellar polarization with wavelength. A Serkowski function cannot be fitted here in view if instrumental effects.

In the context of this work and because of the nature of ESPaDOns data, the quantity of interest is the difference between the value of the polarization in the line and the value in the neighbouring continuum. The detected signal is too weak for this star to study the detailed shape of the polarization line profile. To quantify the difference in polarization between line centre and the neighbouring continuum, a gaussian profile was fitted to each of the lines in $Q / I$ and $U / I$. Table 1 lists the values of the core intensity of these gaussians for the main lines of WR 1.

Let the relative line strength, $R$ be defined as $R=\left(I_{\text {line }}+I_{\text {cont }}\right) / I_{\text {cont }}$, where $I_{\text {line }}$ is the flux in the line only i.e. does not include continuum flux. Also, if the line polarization flux is assumed to be zero, the polarization flux at the position of a spectral line, $Q_{\text {line }}, U_{\text {line }}$ is simply equal to the polarization flux of the continuum, $Q_{\text {cont }}, U_{\text {cont }}$. The difference between the relative polarization at the position of a spectral line and that if the neighbouring continuum is then

$$
\begin{aligned}
& q_{\text {line }}-q_{\text {cont }}=\frac{Q_{\text {line }}}{\left(I_{\text {line }}+I_{\text {cont }}\right)}-\frac{Q_{\text {cont }}}{I_{\text {cont }}}=\frac{Q_{\text {line }}}{R I_{\text {cont }}}-\frac{Q_{\text {cont }}}{I_{\text {cont }}}=\frac{Q_{\text {cont }}}{I_{\text {cont }}}\left(\frac{1}{R}-1\right)=q_{\text {cont }}\left(\frac{1}{R}-1\right), \\
& u_{\text {line }}-u_{\text {cont }}=\frac{U_{\text {line }}}{\left(I_{\text {line }}+I_{\text {cont }}\right)}-\frac{U_{\text {cont }}}{I_{\text {cont }}}=\frac{U_{\text {line }}}{R I_{\text {cont }}}-\frac{U_{\text {cont }}}{I_{\text {cont }}}=\frac{U_{\text {cont }}}{I_{\text {cont }}}\left(\frac{1}{R}-1\right)=u_{\text {cont }}\left(\frac{1}{R}-1\right),
\end{aligned}
$$

where $q$ and $u$ are the relative Stokes parameters. 
Table 1: Linear Polarization Measurements for the Main Spectral Lines of WR 1

\begin{tabular}{lccc}
\hline \hline Line & Q/I & U/I & $I / I_{c}$ \\
& $(\%)$ & $(\%)$ & \\
\hline HeII $\lambda 4542$ & -0.145 & +0.180 & 1.9 \\
NV $\lambda 4603-19$ & -0.181 & +0.216 & 2.4 \\
HeII $\lambda 4686$ & -0.315 & +0.358 & 9.9 \\
HeII $\lambda 4861$ & -0.132 & +0.305 & 2.0 \\
NV $\lambda 4945$ & -0.092 & +0.126 & 1.25 \\
HeII $\lambda 5411$ & -0.169 & +0.252 & 2.7 \\
CIV $\lambda 5804$ & -0.116 & +0.115 & 2.1 \\
HeI $\lambda 5876$ & -0.062 & +0.086 & 1.3 \\
HeII $\lambda 6410$ & -0.101 & +0.122 & 1.2 \\
HeII $\lambda 6560$ & -0.192 & +0.226 & 3.6 \\
HeI $\lambda 6678$ & -0.070 & +0.086 & 1.4 \\
NIV $\lambda 7113$ & -0.244 & +0.167 & 3.5 \\
\hline
\end{tabular}


These equations are exactly the same as those of Schulte-Ladbeck et al. (1991). From them, it can be seen that the slope of a $q_{\text {line }}-q_{\text {cont }}\left(u_{\text {line }}-u_{\text {cont }}\right)$ versus $1 / R$ plot gives $q_{\text {cont }}$ $\left(u_{\text {cont }}\right)$ and that the intercept gives $-q_{\text {cont }}\left(-u_{\text {cont }}\right)$. In Figure 3 , we plot the difference between the relative Stokes parameters in the line and continuum versus the inverse of the relative line strength, $R$. Straight lines were fitted based on $\chi^{2}$ statistics. The values of the slopes and intercepts for both linear Stokes parameters with their associated uncertainties are indicated on the plots.

The final values of $q_{\text {cont }}$ and $u_{\text {cont }}$ are calculated by taking the mean, weighted by the inverse of the square of the uncertainty, of the slope and the intercept (multiplied by -1) of the above plots: $q_{\text {cont }}=0.301 \pm 0.018 \%$ and $u_{\text {cont }}=-0.325 \pm 0.035 \%$. This gives

$$
\begin{gathered}
p_{\text {cont }}=\frac{P_{\text {cont }}}{I_{\text {cont }}}=\sqrt{q_{\text {cont }}^{2}+u_{\text {cont }}^{2}}=0.443 \pm 0.028 \% \\
\theta=\frac{1}{2} \tan ^{-1}\left(u_{\text {cont }} / q_{\text {cont }}\right)=-26.2 \pm 2.6^{\circ}
\end{gathered}
$$

One can wonder what would happen if the assumption that the lines are completely unpolarized was incorrect. In such a case, we would have :

$$
\begin{aligned}
& \left(I_{\text {line }}+I_{\text {cont }}\right) q_{\text {line }}=I_{\text {cont }} q_{\text {cont }}+I_{\text {line }} q_{\text {line }}^{\prime} \\
& \left(I_{\text {line }}+I_{\text {cont }}\right) u_{\text {line }}=I_{\text {cont }} u_{\text {cont }}+I_{\text {line }} u_{\text {line }}^{\prime}
\end{aligned}
$$

where the primed polarization values are those only in the line.

From this follows:

$$
\begin{aligned}
q_{\text {line }} & =\frac{I_{\text {cont }} q_{\text {cont }}+I_{\text {line }} q_{\text {line }}^{\prime}}{I_{\text {line }}+I_{\text {cont }}} \\
u_{\text {line }} & =\frac{I_{\text {cont }} u_{\text {cont }}+I_{\text {line }} u_{\text {line }}^{\prime}}{I_{\text {line }}+I_{\text {cont }}}
\end{aligned}
$$

As $R=I_{\text {cont }} /\left(I_{\text {line }}+I_{\text {cont }}\right)$, the $I_{\text {line }}=I_{\text {cont }}(R-1)$. We then have 


$$
\begin{gathered}
q_{\text {line }}-q_{\text {cont }}=\frac{q_{\text {cont }}}{R}+\frac{I_{\text {cont }}(R-1)}{I_{\text {line }}+I_{\text {cont }}} q_{\text {line }}^{\prime}-q_{\text {cont }} \\
u_{\text {line }}-u_{\text {cont }}=\frac{u_{\text {cont }}}{R}+\frac{I_{\text {cont }}(R-1)}{I_{\text {line }}+I_{\text {cont }}} u_{\text {line }}^{\prime}-u_{\text {cont }}
\end{gathered}
$$

and finally

$$
\begin{gathered}
q_{\text {line }}-q_{\text {cont }}=\left(q_{\text {cont }}-q_{\text {line }}^{\prime}\right) \frac{1}{R}-\left(q_{\text {cont }}-q_{\text {line }}^{\prime}\right)=\left(q_{\text {cont }}-q_{\text {line }}^{\prime}\right)\left(\frac{1}{R}-1\right) \\
u_{\text {line }}-u_{\text {cont }}=\left(u_{\text {cont }}-u_{\text {line }}^{\prime}\right) \frac{1}{R}-\left(u_{\text {cont }}-u_{\text {line }}^{\prime}\right)=\left(u_{\text {cont }}-u_{\text {line }}^{\prime}\right)\left(\frac{1}{R}-1\right)
\end{gathered}
$$

If $q_{\text {line }}^{\prime}$ and $u_{\text {line }}^{\prime}$ are constant for all lines, a plot of $q_{\text {line }}-q_{\text {cont }}\left(u_{\text {line }}-u_{\text {cont }}\right)$ versus $1 / R$ would still give a straight line. However, in view of the well-known stratification of WR stellar winds (e.g. Herald et al. 2000), this is unlikely. The lines formed closer to the base of the wind should have higher polarizations in view of the higher electron densities in these regions. If the situation were that simple, this would lead to a systematic trend in the deviation of the points from the straight-line which assumes zero line polarization. However, it will most likely be more complicated since for axisymmetric winds, the mass-loss and wind velocity become latitude dependent. Also, the star could have a different brightness at the pole and equator. Finally, depending if the ion is a dominant one or not, it may become more or less prominent in the low or high density flow (e.g. Lamers \& Pauldrach 1991; Petrenz \& Puls 2000; Ignace \& Brimeyer 2006). Therefore, the effect of line polarization would likely be to randomly add scatter to the linear relation.

\section{Discussion}

The data presented in this paper represents a new detection of an asymmetric wind of a WR stars using spectropolarimetry. How does this compare with previous observations in 
terms of absolute level of polarization and type of WR star in which it appears and what can be concluded as to the origin of the asymmetry?

Schmidt (1988) published spectropolarimetric observations of several WR stars obtained with the Image-Dissector Scanner spectropolarimeter at Lick Observatory in the early 80s. The original bin size was $\sim 10 \AA$ but the final polarization values were based on bins of 15-20 $\AA$ wide. For WR1, no line depolarization was detected and the final overall value of $P$ given by the author is $P=6.24 \% \pm 0.03 \%$. However this error bar is most certainly the error on the mean taken over the entire wavelength range of their data and is not representative of the scatter in the spectrum. Indeed, an eye estimation from a visual inspection of the $P$ spectrum plot yields (for lack of a better means) a scatter around the mean (on the plot with $10 \AA$ bins, however) which is closer to $2 \sigma \sim 1 \%$ indicating that the error bar on individual bins is closer to $0.5 \%$ instead of $0.03 \%$. This would be more compatible with the results presented in the present work. Indeed, a line depolarization of $0.443 \%$ would have been easily detected if the error bar on each individual wavelength bin would have been $0.03 \%$. On the other hand, such a small value would be lost in the scatter in the plot provided in the paper.

Harries et al. (1998) presented a compilation of 29 WR stars with accurate $(0.05 \%)$ spectropolarimetry and found only 6 that showed line depolarization. With this new detection, there are now seven WR stars for which the line effect has been reported: WR1 (WN4b), WR6 (WN6b), WR16 (WN8h), WR40 (WN8h), WR134 (WN6b), WR136 $(\mathrm{WN6b}(\mathrm{h}))$ and WR137 (WC7pd+O4-5). Of these stars, three, all broad-lined WN stars have been shown to present periodic variations thought to be caused by the presence of CIRs in their wind, WR1 (Chené \& St-Louis 2010), WR 6 (St-Louis et al. 1995; Morel et al. 1997) and WR134 (McCandliss et al. 1994; Morel et al. 1999). WR 136, the only other broad-line WN star, is a special case since line depolarization has been detected only once 
by Whitney et al. (1988). Schmidt (1988), Schulte-Ladbeck (1994) and Harries et al. (1998) also observed this star but made no detection of line depolarization. WR 137, a long-period WC7pd+O9 binary ( $\mathrm{P}=4766$ days $)$ was found to also show photometric variability with a very short 0.83-day period (Lefèvre et al. 2005). Those authors suggest that the short term variability might also be associated with the presence of a CIR, although that has not yet been demonstrated with spectroscopic observations. Finally, the two last stars, WR16 and WR40 are part of the WN8 sub-class which is well-known for showing high levels of variability in photometry, polarimetry and spectroscopy (e.g. Moffat \& Shara 1986; Drissen et al. 1987; Robert et al. 1989; Antokhin et al. 1995; Marchenko et al. 1998).

The seven stars listed above therefore have winds that did not have spherical symmetry at the time the spectropolarimetric observations were obtained. However, the detected continuum polarization is not necessarily constant in time. Indeed, most WR stars are found to show variable continuum polarization. In a series of papers (St.-Louis et al. 1987; Drissen et al. 1987; St.-Louis et al. 1988; Drissen et al. 1989; Robert et al. 1989, 1990; Drissen et al. 1992), the broadband continuum polarization behaviour with time of a sample of WR stars was studied. A correlation between the degree of random, intrinsic scatter in polarization and both the spectral sub-type and wind terminal velocity was found. These two parameters are, however, found to be correlated (e.g. Crowther 2007). Faster-wind WR stars are found to show a lower level of intrinsic variability. The random polarization variability in this case is thought to be a consequence of inhomogeneities in the wind, which is considered to be ubiquitous for this type of outflow (e.g. Lépine \& Moffat 2008). At any given time the blob distribution generates a net polarization level which fluctuates randomly in time because the global blob configuration changes. Several theoretical studies have been carried out on this subject. Initial models by Richardson et al. (1996) (but see also Brown et al. 1995) were unable to reproduce the observed ratio of polarimetric to photometric variability $R=\sigma_{\text {pol }} / \sigma_{\text {phot }} \simeq 0.05$ without invoking very high densities within 
the blobs. 3- $D$ simulations of random (in time and distribution) blob ejection by Li et al. (2000) and Li et al. (2009) including a $\beta$-law for the wind velocity distribution and stellar occultation effects where not only able to reproduce the observed value of $R$ without a very high value of the blob density but also to carry out estimates of quantities such as the $\beta$-law exponent, the number of blobs and the total mass-loss rates in blobs. A similar model was applied to LBVs by Davies et al. (2007). Recent Monte-Carlo simulations by Townsend (2012) using a novel semi-analytic method concludes that the single scattering approximation made in previous models overestimates the mean polarization of optically thick winds such as those of WR stars, even if the clumps themselves are optically thin.

There is no reason to believe that random, intrinsic scatter in polarization cannot happen in a wind that is asymmetric. Therefore, the scatter could be centred on zero linear polarization if the wind is spherically symmetric or on another value if it is not. Of course, one must remove the interstellar polarization beforehand. One might think that with one snapshot observation in spectropolarimetry, it is impossible to conclude if the continuum polarization we are measuring is from a wind asymmetry that is constant in time or if it is due to a random arrangement of blobs in the wind that is generating a residual polarization. However, the level of continuum linear polarization $(\sim 0.5 \%)$ measured for the three broad-line WN stars shown to have CIRs in their wind (WR1, WR6 and WR134) is most likely due to a large-scale structure. Indeed, the levels of scatter generated from random fluctuations ranges from $\sim 0.01-0.04 \%$ for early type WN stars to $\sim 0.08-0.15 \%$ for WN8 stars. This is much smaller than the measured values in those 3 cases. Therefore it is extremely unlikely that the residual polarization measured for these stars stems from a lucky configuration of blobs in the wind. More likely, it is the sign of a large-scale structure in the wind. Such levels of variability can be produced by either a flattened wind (Harries et al. 1998) or a CIR in the wind (Ignace et al. 2009) 
WR136 might be a similar case. Whitney et al. (1988) measured the linear polarization of this star in 10 broad filters from $\sim 4200$ to $8500 \AA$. They found a dip of $\Delta P \sim 0.5 \%$ in one of them centred at $5900 \AA$ ( $100 \AA$ wide). This filter includes the HeI $\lambda 5876$ line and therefore they concluded that the star showed line depolarization. However, this was not observed by 3 other authors in independent observations (see above). One possible explanation is that the line-depolarization for this star or in other words the polarization of its continuum light is a transient phenomenon. If the linear polarization is due to the presence of a CIR for example, it might mean that they occur only occasionally in this star. One supporting observation for CIRs in the wind of this star is the detection of enhanced P Cygni absorption at velocities in excess of the terminal velocity of the wind in the CIV $\lambda 1550$, HeII $\lambda 1640$ and NIV $\lambda 1718$ ultraviolet (UV) P Cygni profiles of this star by St-Louis et al. (1989). The variations were observed over a period of 24 hours in 1987. Two other datasets, one in 1981 and the other in 1982, covering a similar period of time, were found not to show enhanced P Cygni absorption and indeed no variations at all. This type of blue-edge variability is thought to be associated with the presence of CIRs in the wind (Cranmer \& Owocki 1996).

The WC7pd+ O9 binary WR137 shows a modest line linear depolarization of 0.1-0.2\% (Harries et al. 1998, de la Chevrotière et al. submitted). WR+O binaries are well known to show significant levels of linear polarization variability in phase with the orbital period (St.-Louis et al. 1987) as the O-star continuum acts as an asymmetric light source for the free electron distribution in the WR wind. However, in this case, the $13.05 \mathrm{yr}$ orbit (Williams et al. 2001) is much too large for such an effect to take place. Therefore as already mentioned by Harries et al. (1998) the continuum polarization is more likely intrinsic to the WR wind. The random broadband polarization variability reported by Robert et al. (1989) for this star is of a similar level. Therefore, it is possible that the continuum polarization of the WR star in this system is simply due to blobs in the wind. However, 
one must also consider that because this is a $\mathrm{WR}+\mathrm{O}$ binary, the relative polarization measurements of the line depolarization are diluted because although the polarization is likely to occur in the wind of one star, we are dividing that polarized flux by the total light of the two stars, thereby diluting the line-depolarization. The original linear polarization in the wind is therefore most certainly higher and might be caused by a global asymmetry in the wind. This result is possibly supported by the work of Lefèvre et al. (2005) who found a very short $0.83 \mathrm{~d}$ period in small-amplitude variations of the absorption throughs of the CIV $\lambda \lambda 5802 / 5812$ and HeI $\lambda 5876$ P Cygni profiles, which they associate with CIRs or non-radial pulsations. If it were CIRs in the WR wind, it could explain the continuum polarization level observed by Harries et al. (1998).

Finally, the line depolarization measured for the two WN8 stars WR16 and WR40 (Vink et al. 2011) on the other hand is very small, of the order of $\sim 0.1 \%$. This could very well be a random polarization level due to blobs, particularly because the scatter in polarization is very high for these WN8 type stars.

\section{Conclusion}

In summary, of the seven stars showing line depolarization and therefore having some level of continuum polarization, three (WR1, WR6 \& WR134) and possibly four (including WR136) have quite a large amplitude of $\sim 0.5 \%$ that can be explained either by a flattened wind or the presence of large-scale structure such as CIRs. For WR1, WR6 and WR134, the presence of CIRs in the wind is supported by kinematic evidence from periodic variations in optical emission lines. Therefore this hypothesis is preferred. For WR136, the phenomenon seems to be more elusive and needs to be confirmed. These four stars are all broad-line

early WN stars. Based on the work of Hamann et al. (1993), Smith et al. (1996) introduced the $b$ subscript (together with others) as an extension of the classical WR star classification 
system to distinguish those that have a HeII $\lambda 5411$ equivalent width greater than $40 \AA$. The usefulness of this was questioned by Conti (1999) because it was found that the observed distribution of line width did not have a sufficiently clear delimitation at the $40 \AA$ level (but see Smith et al. 2000). So it is not clear if these stars have a peculiar characteristic that can help explain the presence of CIRs in their wind or if the presence of CIRs in these three stars with a WNb classification is just a simple coincidence. One difference is that stars with the WNb classification are never found to have hydrogen while those that are simply classified WN sometimes are found to have hydrogen and sometimes not. A final point to consider is the recent strong correlation found by Vink et al. (2011) and Gräfener et al. (2012) between line depolarization in WR stars and the presence of an ejected nebula. As ejected nebula are predicted to last only for $\sim 10^{5}$ years (van Marle et al. 2005), these authors associated line depolarization with youth in the WR phase. This might indicate that CIRs only occur in WR winds when they have just recently reached this evolutionary phase.

Stars that have CIRs in their winds are extremely useful because if the structures are pinned to their surfaces, the periods of the spectroscopic variations they generate together with an estimate of the stars' radii allows us to measure the rotation velocities of the stars, which cannot be obtained by regular means as there are no photospheric lines in the spectrum. However, CIRs are structures that appear and dissipate on an unknown timescale which leads to epoch-dependant variations. Therefore, they are extremely difficult to identify. If one can establish a link between line depolarization of WR stars and the presence of a CIR, this can provide an easier means of identifying them. Although detailed spectroscopic follow-up observations would still be required to determine the rotation period, this would certainly represent a significant improvement on the present situation.

First, NSL would like to thank the referee, Rico Ignace for a thorough evaluation of 
the manuscript, including many constructive comments that led to the improvement of this paper. NSL also thanks Nadine Manset from the CFHT for continuous support in data reduction issues and Anthony F.J. Moffat and Noel Richardson for comments on the manuscript. NSL also acknowledges financial support from the Natural Science and Engineering Research Council (NSERC) of Canada.

Facilities: CFHT 


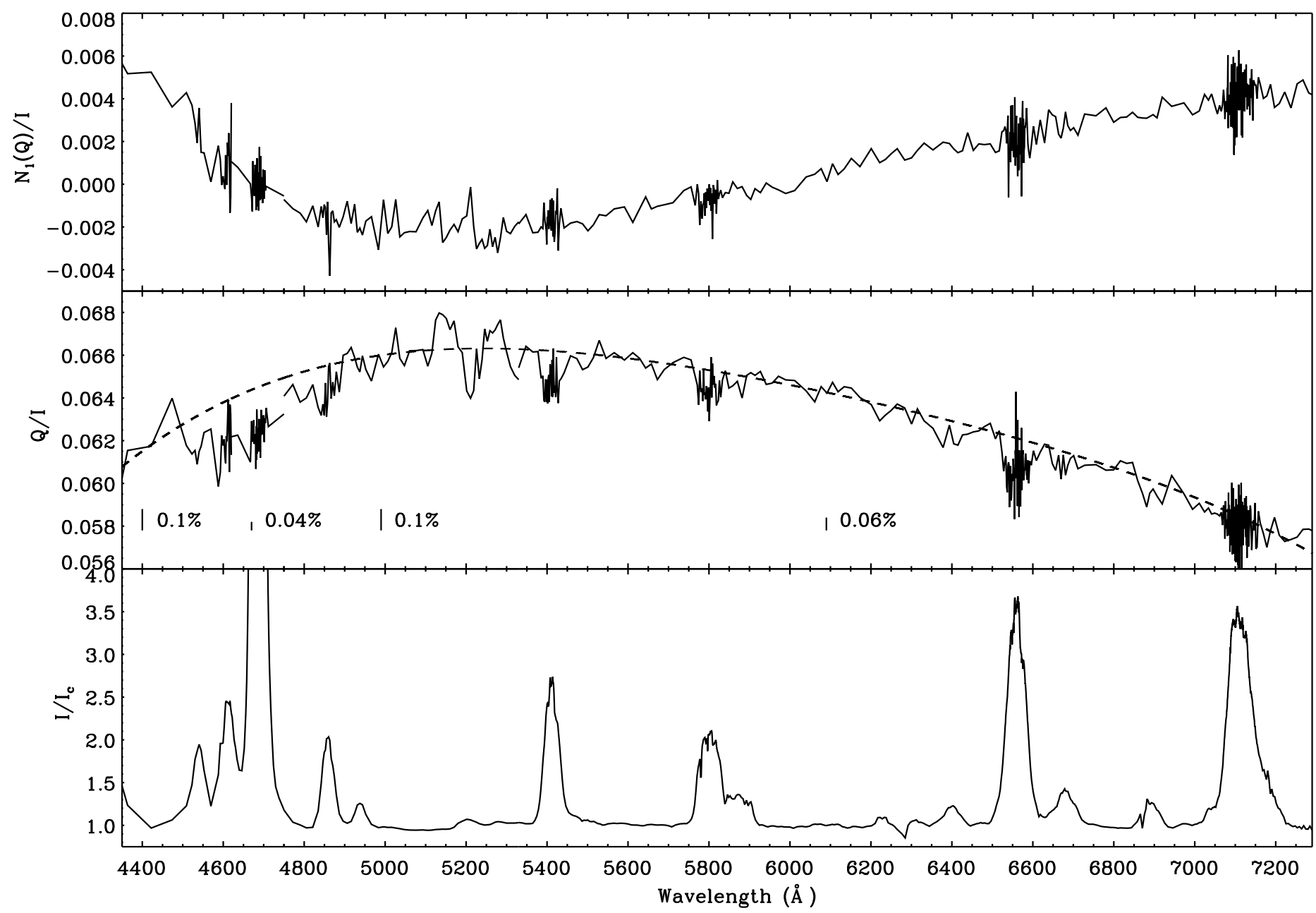

Fig. 1.- Relative Stokes parameter $I / I_{c}$ (bottom), $Q / I$ (middle) and check spectra $N_{1}(Q) / I$ (top) for the WR star WR 1. Binned $2 \sigma$ error bars are variable along the spectra and are indicated in the middle panel. 


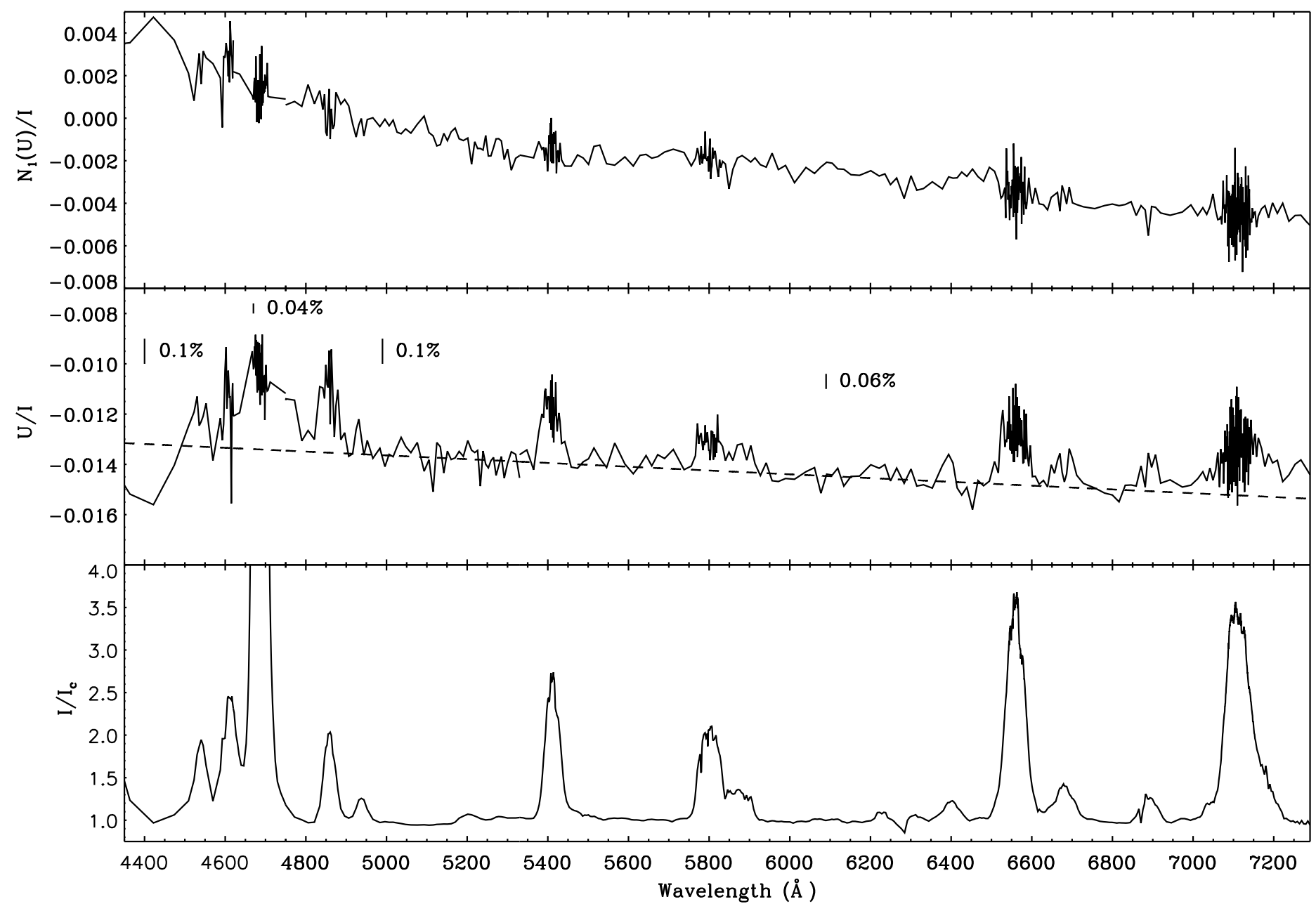

Fig. 2.- Relative Stokes parameter $I / I_{c}$ (bottom), $U / I$ (middle) and check spectra $N_{1}(U) / I$ (top) for the WR star WR 1. Binned $2 \sigma$ error bars are variable along the spectra and are indicated in the middle panel. 

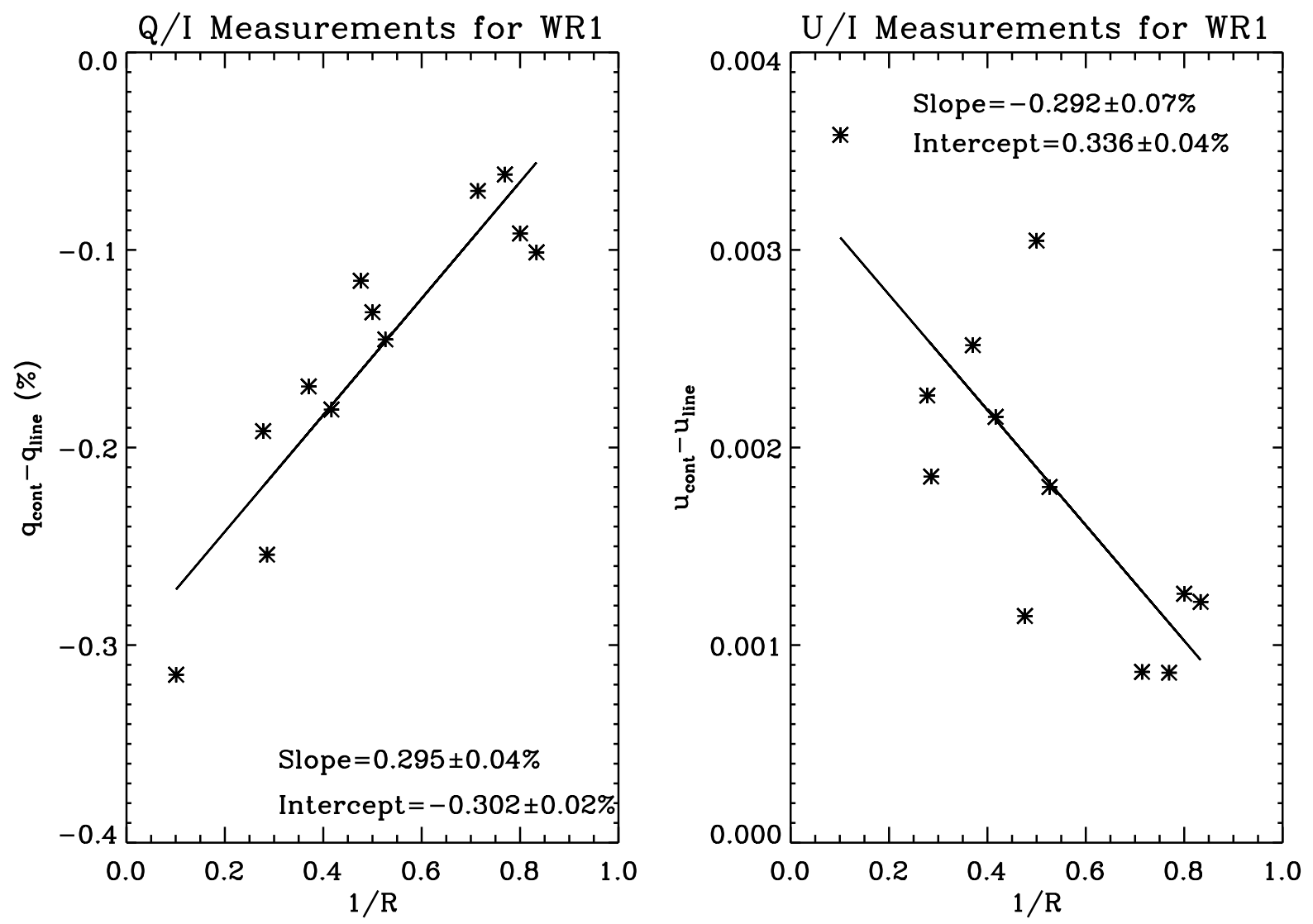

Fig. 3. - $q_{\text {line }}-q_{\text {cont }}$ and $u_{\text {line }}-u_{\text {cont }}$ versus $1 / \mathrm{R}$ plots for WR1. The slope and intercept of the straight line fitted to the data give the value of the continuum polarization of the star. 


\section{REFERENCES}

Antokhin, I., Bertrand, J.-F., Lamontagne, R., Moffat, A. F. J., \& Matthews, J. 1995, AJ, 109,817

Bouret, J.-C., Lanz, T., \& Hillier, D. J. 2005, A\&A, 438, 301

Brott, I., de Mink, S. E., Cantiello, M., et al. 2011, A\&A, 530, A115

Brown, J. C., Richardson, L. L., Antokhin, I., et al. 1995, A\&A, 295, 725

Chené, A.-N., \& St-Louis, N. 2010, ApJ, 716, 929

Chiosi, C., \& Maeder, A. 1986, ARA\&A, 24, 329

Conti, P. S. 1999, New A, 4, 489

Cranmer, S. R., \& Owocki, S. P. 1995, ApJ, 440, 308

-. 1996, ApJ, 462, 469

Crowther, P. A. 2007, ARA\&A, 45, 177

Davies, B., Vink, J. S., \& Oudmaijer, R. D. 2007, A\&A, 469, 1045

de la Chevrotière, A., St-Louis, N., Moffat, A. F. J., \& the MiMeS Collaboration. 2013, ApJ, 764, 171

Dessart, L., \& Chesneau, O. 2002, A\&A, 395, 209

Donati, J.-F., Semel, M., Carter, B. D., Rees, D. E., \& Collier Cameron, A. 1997, MNRAS, 291,658

Drissen, L., Robert, C., Lamontagne, R., et al. 1989, ApJ, 343, 426

Drissen, L., Robert, C., \& Moffat, A. F. J. 1992, ApJ, 386, 288 
Drissen, L., St.-Louis, N., Moffat, A. F. J., \& Bastien, P. 1987, ApJ, 322, 888

Gayley, K. G., \& Ignace, R. 2010, ApJ, 708, 615

Gräfener, G., Vink, J. S., Harries, T. J., \& Langer, N. 2012, A\&A, 547, A83

Hamann, W.-R., \& Koesterke, L. 1998, A\&A, 335, 1003

Hamann, W. R., Koesterke, L., \& Wessolowski, U. 1993, A\&A, 274, 397

Harries, T. J., Hillier, D. J., \& Howarth, I. D. 1998, MNRAS, 296, 1072

Herald, J. E., Schulte-Ladbeck, R. E., Eenens, P. R. J., \& Morris, P. 2000, ApJS, 126, 469

Howarth, I. D., \& Prinja, R. K. 1989, ApJS, 69, 527

Ignace, R., \& Brimeyer, A. 2006, MNRAS, 371, 343

Ignace, R., Hubrig, S., \& Schöller, M. 2009, AJ, 137, 3339

Lamers, H. J. G., \& Pauldrach, A. W. A. 1991, A\&A, 244, L5

Langer, N. 2012, ARA\&A, 50, 107

Lefèvre, L., Marchenko, S. V., Lépine, S., et al. 2005, MNRAS, 360, 141

Lépine, S., \& Moffat, A. F. J. 1999, ApJ, 514, 909

-. 2008, AJ, 136, 548

Li, Q., Brown, J. C., Ignace, R., Cassinelli, J. P., \& Oskinova, L. M. 2000, A\&A, 357, 233

Li, Q.-K., Cassinelli, J. P., Brown, J. C., \& Ignace, R. 2009, Research in Astronomy and Astrophysics, 9, 558

Maeder, A. 1981, A\&A, 102, 401 
-. 1999, A\&A, 347, 185

Maeder, A., \& Meynet, G. 2000, ARA\&A, 38, 143

-. 2004, A\&A, 422, 225

Marchenko, S. V., Moffat, A. F. J., Eversberg, T., et al. 1998, MNRAS, 294, 642

McCandliss, S. R., Bohannan, B., Robert, C., \& Moffat, A. F. J. 1994, Ap\&SS, 221, 155

Meynet, G., Eggenberger, P., \& Maeder, A. 2011, A\&A, 525, L11

Meynet, G., \& Maeder, A. 2000, A\&A, 361, 101

Meynet, G., Maeder, A., Schaller, G., Schaerer, D., \& Charbonnel, C. 1994, A\&AS, 103, 97

Moffat, A. F. J. 1982, in IAU Symposium, Vol. 99, Wolf-Rayet Stars: Observations, Physics, Evolution, ed. C. W. H. De Loore \& A. J. Willis, 263-273

Moffat, A. F. J. 1983, in Wolf-Rayet stars: Progenitors of supernovae?, ed. M.-C. Lortet \& A. Pitault, 13

Moffat, A. F. J., Drissen, L., Lamontagne, R., \& Robert, C. 1988, ApJ, 334, 1038

Moffat, A. F. J., \& Shara, M. M. 1986, AJ, 92, 952

Morel, T., St-Louis, N., \& Marchenko, S. V. 1997, ApJ, 482, 470

Morel, T., Marchenko, S. V., Eenens, P. R. J., et al. 1999, ApJ, 518, 428

Mullan, D. J. 1986, A\&A, 165, 157

Nugis, T., Crowther, P. A., \& Willis, A. J. 1998, A\&A, 333, 956

Petrenz, P., \& Puls, J. 2000, A\&A, 358, 956 
Richardson, L. L., Brown, J. C., \& Simmons, J. F. L. 1996, A\&A, 306, 519

Robert, C., Moffat, A. F. J., Bastien, P., Drissen, L., \& St.-Louis, N. 1989, ApJ, 347, 1034

Robert, C., Moffat, A. F. J., Bastien, P., St.-Louis, N., \& Drissen, L. 1990, ApJ, 359, 211

Schmidt, G. D. 1988, Spectropolarimetry as a probe of the structure of Wolf-Rayet envelopes, ed. G. V. Coyne, A. M. Magalhaes, A. F. Moffat, R. E. Schulte-Ladbeck, \& S. Tapia, 641-654

Schulte-Ladbeck, R. E. 1994, Ap\&SS, 221, 347

Schulte-Ladbeck, R. E., Nordsieck, K. H., Taylor, M., et al. 1991, ApJ, 382, 301

Semel, M., \& Li, J. 1996, Sol. Phys., 164, 417

Serkowski, K., Mathewson, D. S., \& Ford, V. L. 1975, ApJ, 196, 261

Smith, L. F., Shara, M., \& Moffat, A. 2000, New A, 5, 423

Smith, L. F., Shara, M. M., \& Moffat, A. F. J. 1996, MNRAS, 281, 163

St-Louis, N., Chené, A.-N., Schnurr, O., \& Nicol, M.-H. 2009, ApJ, 698, 1951

St-Louis, N., Dalton, M. J., Marchenko, S. V., Moffat, A. F. J., \& Willis, A. J. 1995, ApJ, 452, L57

St.-Louis, N., Drissen, L., Moffat, A. F. J., Bastien, P., \& Tapia, S. 1987, ApJ, 322, 870

St.-Louis, N., Moffat, A. F. J., Drissen, L., Bastien, P., \& Robert, C. 1988, ApJ, 330, 286

St-Louis, N., Smith, L. J., Stevens, I. R., et al. 1989, A\&A, 226, 249

Townsend, R. 2012, in American Institute of Physics Conference Series, Vol. 1429, American Institute of Physics Conference Series, ed. J. L. Hoffman, J. Bjorkman, \& B. Whitney, 278-281 
van Marle, A. J., Langer, N., \& García-Segura, G. 2005, A\&A, 444, 837

Vink, J. S., Gräfener, G., \& Harries, T. J. 2011, A\&A, 536, L10

Vreux, J.-M. 1985, PASP, 97, 274

Whitney, B. A., Schulte-Ladbeck, R. E., Aspin, C., et al. 1988, in Bulletin of the American Astronomical Society, Vol. 20, Bulletin of the American Astronomical Society, 1013

Williams, P. M., Kidger, M. R., van der Hucht, K. A., et al. 2001, MNRAS, 324, 156

Woosley, S. E., Langer, N., \& Weaver, T. A. 1993, ApJ, 411, 823

Yoon, S.-C., Dierks, A., \& Langer, N. 2012, A\&A, 542, A113 